\title{
Who Benefits From a Defibrillator-Balancing the Risk of Sudden Versus Non-sudden Death
}

\author{
Simon A. S. Beggs ${ }^{1} \cdot$ Roy S. Gardner ${ }^{2} \cdot$ John J. V. McMurray ${ }^{1}$ \\ Published online: 10 November 2018 \\ (C) The Author(s) 2018
}

\begin{abstract}
Purpose of Review Treatment with a defibrillator can reduce the risk of sudden death by terminating ventricular arrhythmias. The identification of patient groups in whom this function reduces overall mortality is challenging. In this review, we summarise the evidence for who benefits from a defibrillator.

Recent Findings Recent evidence suggests that contemporary pharmacologic and non-defibrillator device therapies are altering the potential risks and benefits of a defibrillator.

Summary Who benefits from a defibrillator is determined by both the risk of sudden death and the competing risk of other, nonsudden causes of death. The balance of these risks is changing, which calls into question whether historic evidence for the use of defibrillators remains robust in the modern era.
\end{abstract}

Keywords Defibrillator $\cdot \mathrm{ICD} \cdot$ Sudden death $\cdot$ Competing risk

\section{Introduction}

In 1980, Mirowski et al. reported the first three patients treated with an automatic implantable defibrillator (AID) [1]. This early AID was a 'shock box', delivering only high-voltage defibrillation therapy. Later devices evolved to include a pacing function and are now usually described as implantable cardioverter defibrillators (ICDs). The randomised controlled trials (RCTs) that followed examined the efficacy of ICD therapy in populations targeted for their high incidence of sudden cardiac death (SCD). Specifically, these were patients with prior cardiac arrest, ventricular arrhythmias (VA) or myocardial infarction (MI), or the presence of coronary artery disease (CAD), left ventricular systolic dysfunction (LVSD), heart failure or some combination of these problems. Incrementally, these RCTs established our current understanding of which patients are more, or less, likely to benefit from an ICD. The importance of several major themes that

This article is part of the Topical Collection on Devices

John J. V. McMurray

John.McMurray@glasgow.ac.uk

1 British Heart Foundation Cardiovascular Research Centre, University of Glasgow, Glasgow, Scotland

2 Golden Jubilee National Hospital, Clydebank, Scotland progressively emerged from these trials must be recognised if a thorough examination of this evidence is undertaken. These include the role of competing risks of death, appropriate trial design-including length of follow-up and the selection of a suitable primary endpoint - the technical evolution of the defibrillator, and incremental advances in prognostic pharmacologic and additional device therapy.

\section{Who Benefits From a Secondary Prevention Defibrillator?}

The benefit of an ICD as a secondary prevention treatment was examined in three major prospective, multi-centre RCTs. Summary characteristics of these studies are presented in Table 1. The first published and largest study was the Antiarrhythmics Versus Implantable Defibrillators (AVID) trial [2•]. Patient eligibility in AVID required one of the following: (1) resuscitation from ventricular fibrillation (VF), (2) syncope with sustained ventricular tachycardia (VT) or (3) sustained VT with significant associated symptoms and left ventricular ejection fraction (LVEF) $\leq 40 \%$. AVID enrolled 1016 patients, randomising them to treatment with an ICD or antiarrhythmic drugs (AADs), primarily amiodarone. Over a mean follow-up of 18 months, there were 80 and 122 deaths from any cause in the ICD and AAD arms, respectively 


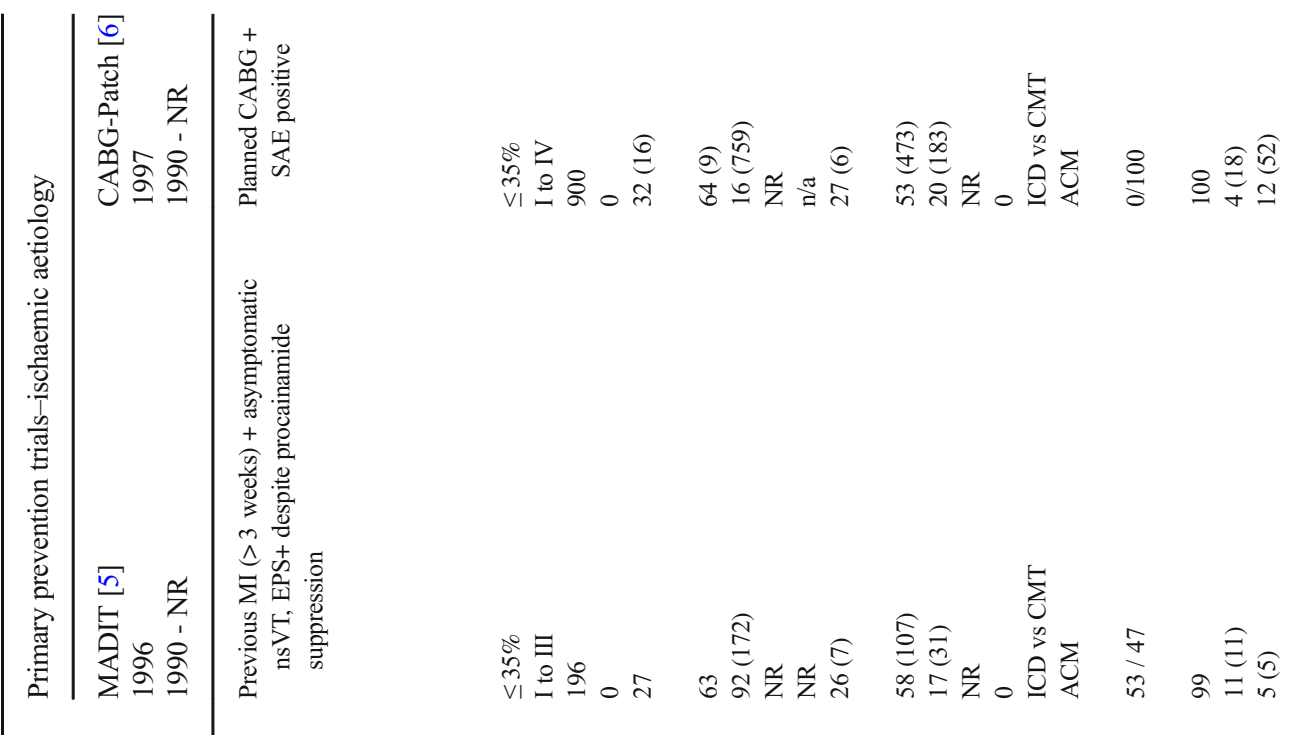

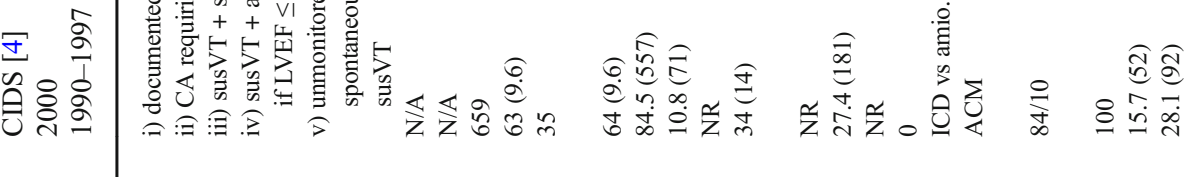<smiles>[CH][CH]C</smiles>

$\underset{\infty}{\infty}$

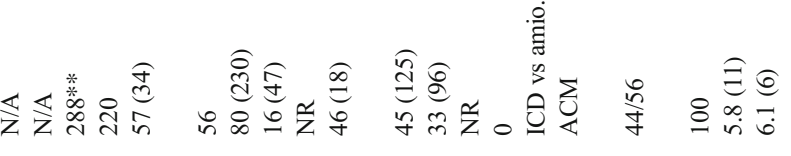




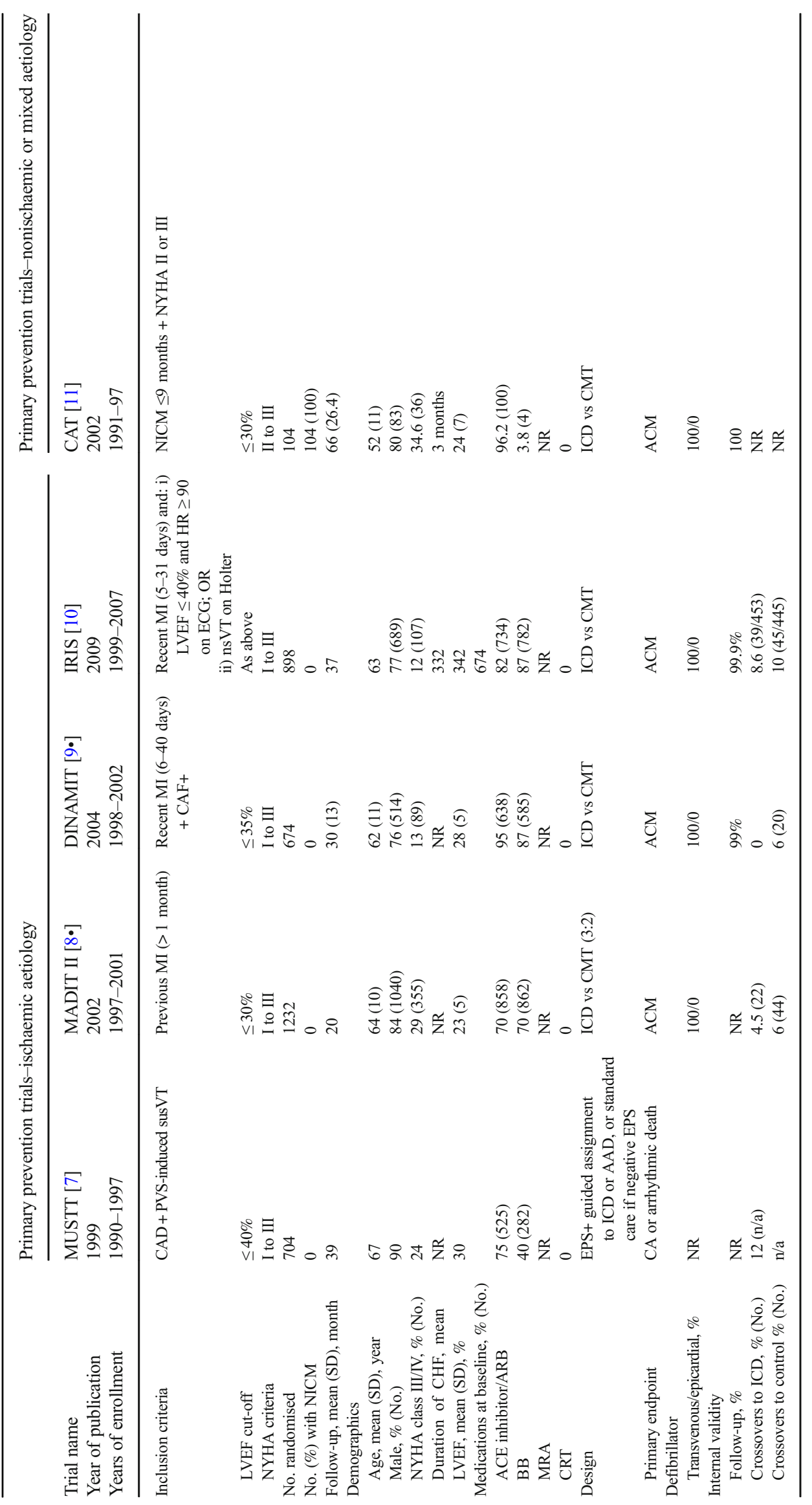




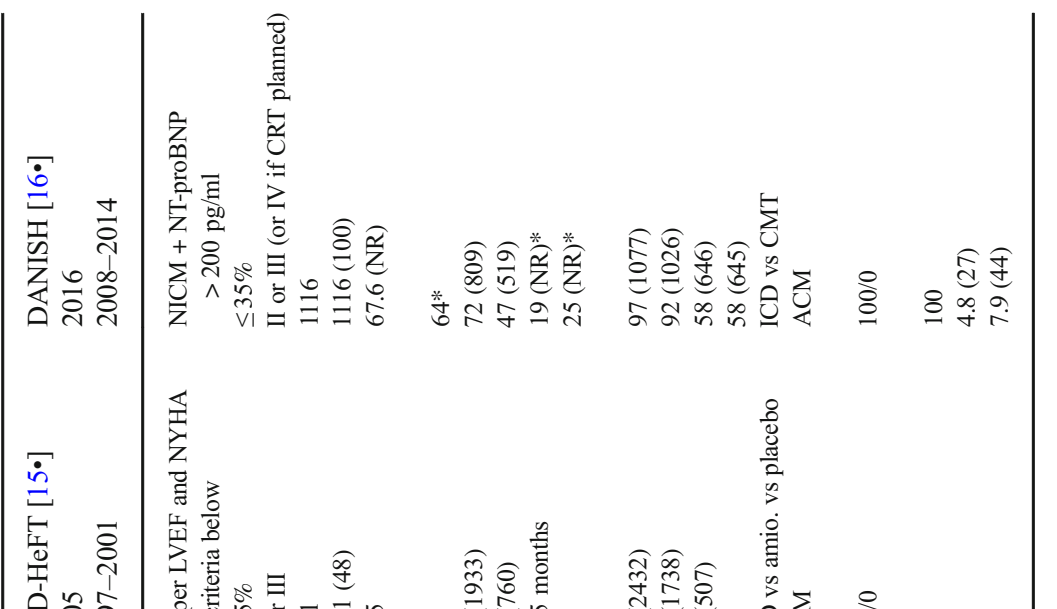

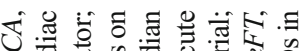

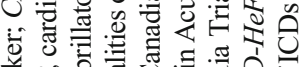

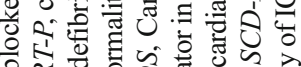

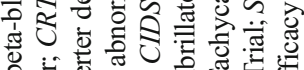

品

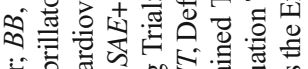

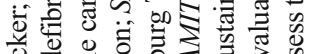

年

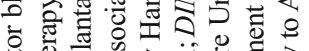

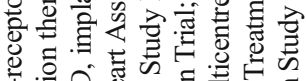

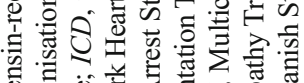

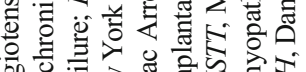

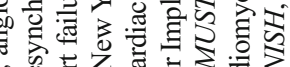

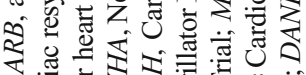

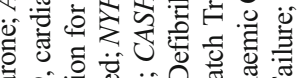

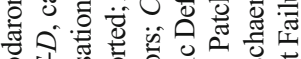

은. 造

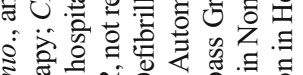

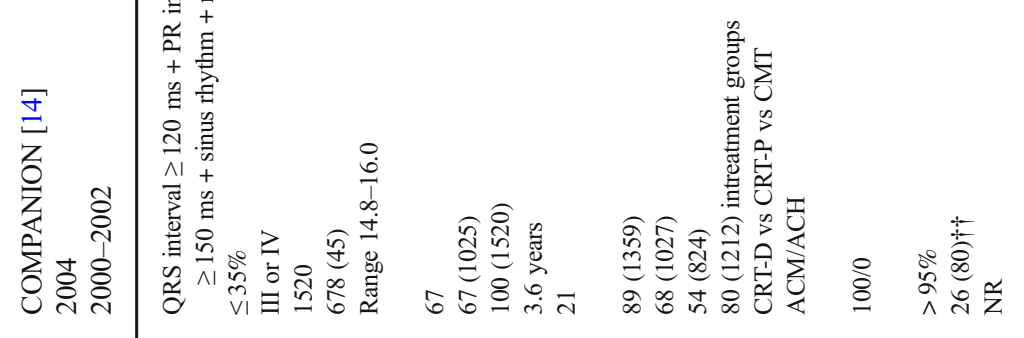

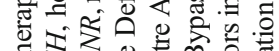

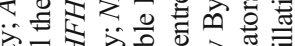

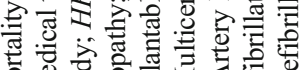

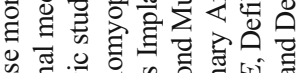

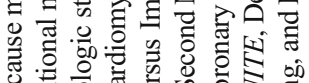

न

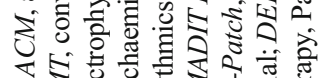

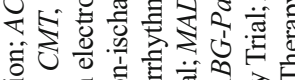

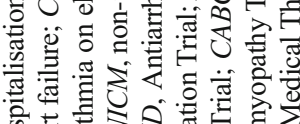

क्ष

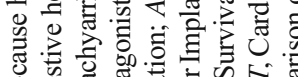

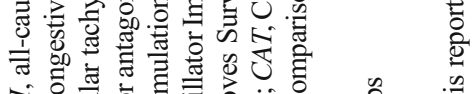

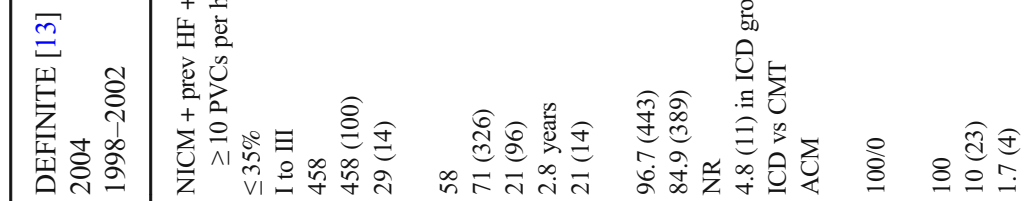

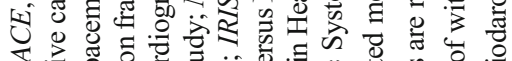

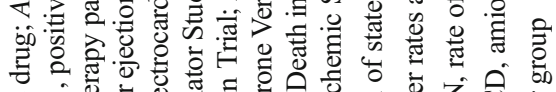

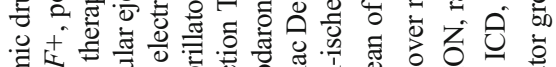

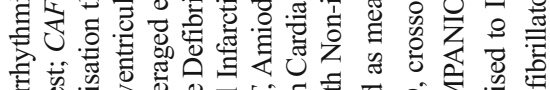

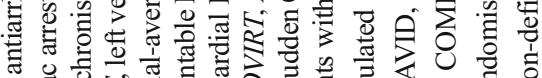

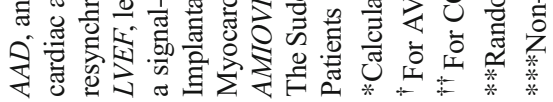


(hazard ratio [HR] 0.62, 95\% confidence intervals [CI] 0.47$0.81, p<0.02)$. This reduction in all-cause mortality (ACM) was driven by a reduction in SCD (HR $0.43,95 \%$ CI $0.27-$ 0.66). The Cardiac Arrest Study Hamburg (CASH) trial enrolled $346^{1}$ patients resuscitated from cardiac arrest due to VT/ $\mathrm{VF}$, randomising them to receive an ICD or one of three AADs: amiodarone, metoprolol or propafenone [3]. Finally, the Canadian Implantable Defibrillator Study (CIDS) recruited 659 patients who had experienced VT/VF associated with one of five eligible clinical presentations (see Table 1) [4]. Patients were randomised to an ICD or amiodarone in a 1:1 ratio. Neither CASH nor CIDS demonstrated a significant reduction in ACM with ICD therapy, although treatment with a defibrillator was associated with a lower risk of SCD in CASH (HR 0.32, 95\%CI 0.15-0.69).

One thousand eight hundred sixty-six participants from AVID, CIDS and CASH were subsequently combined in a patient-level meta-analysis evaluating the effects of randomisation to an ICD versus amiodarone or sotalol (thus excluding the 97 patients allocated to metoprolol in CASH) [17•]. Treatment with an ICD conferred a lower risk of ACM (HR $0.73,95 \%$ CI $0.60-0.87, p<0.001$ ), with this effect being driven by a reduction in SCD (HR 0.49, 95\%CI 0.36-0.67, $p<0 \cdot 001$ ). There was no significant difference in non-sudden mortality between the groups. The treatment effect of an ICD was consistent across the three trials $(p$ value for interaction $=$ 0.306), suggesting that CIDS and CASH provide supportive evidence for, rather than militating against, the survival benefit seen in AVID. Another meta-analysis of the secondary prevention trials which included an additional small Dutch study estimated a relative risk of ACM of $0.75,(95 \%$ CI $0.64-0.87$, $p=0 \cdot 0002)$ [18].

These trials form the basis for international guideline recommendations $[19,20]$ for secondary prevention ICDs, including those from the European Society of Cardiology (ESC) and a combined guideline from the American College of Cardiology (ACC), American Heart Association (AHA) and the Heart Rhythm Society (HRS). Selected recommendations from these guidelines are summarised and contrasted in Fig. 1.

As to precisely who benefits from a secondary prevention ICD, there is little more to add. Exploration of subgroups in the first meta-analysis described above found no interaction between treatment effect and index VA, prior MI, nonischemic aetiology, coronary artery disease, New York Heart Association (NYHA) functional class or coronary artery bypass at baseline. However, two significant subgroup interactions were identified. First, patients treated with an ICD during

\footnotetext{
${ }^{1}$ Assignment to propafenone was terminated early due to increased mortality after 58 patients had been randomised to this arm, with results thus reported for the remaining 288 patients in the defibrillator, amiodarone and metoprolol arms.
}

the 'epicardial era' - defined as ending on 1st July 1991 derived less benefit from a defibrillator than those randomised after this date, who largely received transvenous systems ( $P$ for interaction $=0.029$ ). Although this effect is likely confounded by other advances in management pre- and post1991, the proposition that transvenous ICDs should be superior to epicardial systems - which require a thoracotomy - is patently plausible. Today, epicardial ICDs are seldom implanted. Second, patients with LVEF $\leq 35 \%$ derived significantly more benefit from a defibrillator than those with LVEF $>35 \%$ ( $P$ for interaction $=0.011$ ), in whom there was a nonsignificant trend towards harm (HR 1.2, 95\% CI 0.81-1.76). The CASH trial-in which more than half of patients received an epicardial system - enrolled a population with a significantly higher mean LVEF than AVID or CIDS (46\% vs $31 \%$ and $34 \%$ respectively). It is therefore possible that the lower efficacy of an ICD detected in the LVEF $>35 \%$ group was actually due to more frequent epicardial ICD use in this population. In any case, subgroup analyses must obviously be treated with caution: consequently, international guideline recommendations for secondary prevention ICDs do not differentiate according to LVEF, and further evidence from prospective studies would be required before this would change.

A final point of interest is that, in this meta-analysis, the incremental separation over time of the Kaplan-Meier curves for arrhythmic death contrasts with the lack of progressive divergence between the curves for ACM, which initially separates before starting to converge after 4 years. Although this suggested that the benefit of an ICD might wane over a longer period, further insight is curtailed by the relatively short follow-up in AVID and CIDS.

\section{Who Benefits From a Primary Prevention Defibrillator?}

\section{Acute MI: Late Implantation of an ICD}

The quest to identify further patient groups which might benefit from an ICD led next to primary prevention trials in patients with a history of acute myocardial infarction (AMI). At the time - approximately three decades ago-4 to 5-year mortality following hospital discharge after AMI was $\geq 20 \%$ amongst patients with LVSD [21-23], with SCD accounting for roughly one third of late mortality $[21,24]$. Seminal amongst these new trials was the Second Multicentre Automatic Defibrillator Implantation Trial (MADIT II) [8•]. Although three prior RCTs [5-7] had examined the benefit of an ICD in patients with CAD and/or MI, all had required the presence of VT and/or an abnormal signal-averaged electrocardiogram (SAECG) (see Table 1). Inclusion criteria for the original Multicentre Automatic Defibrillator Implantation Trial 


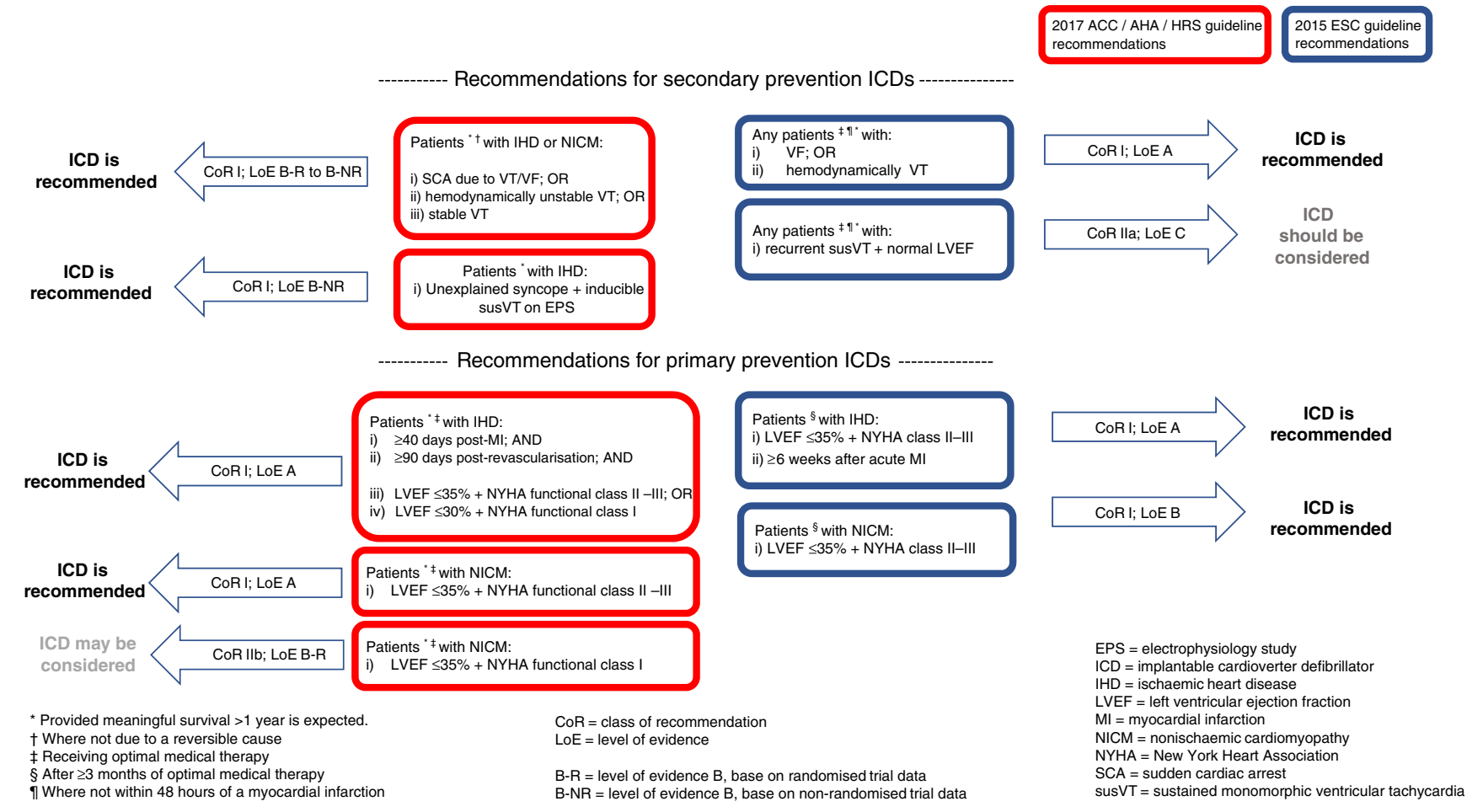

Fig. 1 Selected international guideline recommendations for implantable cardioverter defibrillators

(MADIT I), for example, had included not merely LVSD and a prior AMI, but also asymptomatic non-sustained VT and inducible, non-suppressible VT on an electrophysiology (EP) study. These predecessor trials were thus less pragmatic and less broadly applicable than MADIT II, in which eligible patients had experienced an AMI 1 month or more prior to enrolment (although in three-quarters of patients the gap was 18 months prior or longer, as shall be discussed later), had an LVEF $\leq 30 \%$, and had not undergone coronary revascularisation within the preceding 3 months. Participants were assigned in a 3:2 ratio to receive either an ICD $(n=742)$ or conventional medical therapy $(\mathrm{CMT})(n=490)$, amongst whom only $10 \%$ were prescribed amiodarone.

During a mean follow-up of 20 months, the rates of ACM were $14.2 \%$ and $19.8 \%$ in the ICD and CMT groups respectively, with an ICD conferring a $31 \%$ relative reduction in the risk of death (HR $0.69,95 \%$ CI $0.51-$ $0.93, p=0.016$ ). The effect of ICD therapy on survival did not vary according to any of the subgroups analysed, including those defined by age, sex, LVEF, NYHA class, QRS duration, diabetes, left bundle-branch block or atrial fibrillation. The survival benefit was entirely due to a reduction in $\mathrm{SCD}$, consistent with the plausible benefit of a defibrillator (HR 0.33 , 95\% CI $0.20-0.53$, $p<0.0001$ ) [25]. Notably, although the cumulative hazard curves reflecting SCD separated early, those for ACM diverged only after $\sim 9$ months. This effect appears to have been driven by a trend towards increased non- sudden cardiac death within the ICD group, especially during the first year, although this did not reach statistical significance. There was also a trend to new or worsening $\mathrm{HF}$ in the ICD group.

\section{Acute MI: Early Implantation of an ICD}

Patients within 1 month of an AMI were excluded from MADIT II. Two subsequent RCTs-the Defibrillator in Acute Myocardial Infarction Trial (DINAMIT) and Immediate Risk Stratification Improves Survival (IRIS) trial - thus sought to examine the effect of an ICD in patients following a recent AMI (6-40 days and 531 days post-MI respectively) $[9 \cdot, 10]$. Outline characteristics of both trials are presented in Table 1. Eligibility criteria for DINAMIT included LVEF $\leq 35 \%$ and impaired cardiac autonomic function, with patients randomised to medical therapy with $(n=332)$ or without $(n=342)$ a defibrillator. After an average follow-up of 30 months, there was a $58 \%$ lower risk of SCD in the ICD group when compared to the control group (HR $0.42,95 \%$ CI $0.22-0.83, p=0.009$ ), with 12 and 29 sudden deaths respectively in these groups. An ICD did not confer an overall mortality benefit however, due to a significant increase in non-sudden cardiac deaths in the ICD group (HR 1.75, 95\%CI $1.11-2.76, p=0.02$ ). IRIS required eligible patients to have $\mathrm{LVEF} \leq 40 \%$ and a heart rate $\geq 90$ beats per minute or non-sustained VT on Holter monitor, randomising them to medical therapy 
with $(n=445)$ or without $(n=453)$ a defibrillator. During an average follow-up of 37 months, there was a lower risk of sudden death with an ICD (HR 0.55, 95\% CI $0.31-1.00, p=0.049)$ that, as in DINAMIT, was offset by an increase in non-sudden cardiac death amongst ICD recipients (HR 1.92, 95\%CI 1.29-2.84, $p=0.001)$. Resultantly, there was no difference in ACM between the two groups.

This increase in non-sudden death associated with an ICD in DINAMIT and IRIS is partially explained by the competing risks posed by multiple potential causes of death. In the ICD groups, the reduction in SCD associated with a defibrillator led to a larger pool of survivors at risk of non-sudden death when compared to the control groups. That is, treatment with an ICD may have merely switched the mode of death from arrhythmic to non-arrhythmic (presumably deaths due to ischaemia or heart failure since the difference in non-sudden events was driven by cardiac causes). This is why ACMrather than $\mathrm{SCD}$ - is the more appropriate primary endpoint for a trial examining the benefit of a defibrillator. ${ }^{2}$ Of course, this principle is true of other ICD trials, yet in several increases in non-sudden death were not sufficiently great to offset the concomitant reductions in SCD afforded by an ICD. For example, non-sudden cardiac death increased in the ICD arm of MADIT II (4.3\% of deaths in the control group, compared to $5.8 \%$ of deaths in the ICD group) without nullifying the overall survival benefit of an ICD.

Closer inspection of these trials is instructive. In both DINAMIT and IRIS, clinical events were classified by adjudication committees blinded to treatment assignment; there were few significant differences between the ICD and control group characteristics, and neither trial experienced high crossover rates between treatments. Artefact was therefore unlikely to have substantially contributed to the results. The first major difference in inclusion criteria between DINAMIT/IRIS and other primary prevention trials carried out in patients with ischemic heart disease (IHD) was the use of autonomic function and heart rate criteria in the former (depressed heart-rate variability or elevated 24-h heart rate in DINAMIT and elevated heart rate or non-sustained VT in IRIS). Subsequent research suggests that these phenomena are associated with an increased risk of ACM rather than specifically arrhythmic SCD $[26,27]$. Thus, whereas the investigators had intended these criteria to select participants at particularly high risk of arrhythmic death, they may have inadvertently enriched the study populations with patients also at high risk of non-sudden cardiac death, thus increasing the probability that patients 'saved' from arrhythmic death by an ICD would nevertheless die from an alternative cardiac cause soon after.

\footnotetext{
${ }^{2}$ It was due to this realisation that, midway through the CIDS trial, the Steering Committee revised the primary outcome measure from arrhythmic death to ACM
}

The second major difference in inclusion criteria between DINAMIT/IRIS and other primary prevention trials performed in IHD populations was the temporal proximity to index AMI. This temporal difference is more substantial than might be assumed a priori from inclusion criteria. For example, although MADIT II ostensibly recruited patients who were at least 1 month remote from an AMI, in reality, this period was 81 months on average, and greater than 6 months in nearly $90 \%$ of patients [28]. The subpopulation of DINAMIT and IRIS patients who died during the early postAMI period were thus not meaningfully represented in MADIT II. This survivorship bias is important because this cohort is likely to have had a particularly vulnerable cardiac substrate, susceptible not merely to ventricular arrhythmias but to other causes of cardiac death. By comparison, it may be presumed that patients in other primary prevention RCTs had more compensated, remodelled hearts that were susceptible to scar-related VAs but at relatively lower competing risk of imminent reinfarction or heart failure. Substantiating this, a subgroup analysis of MADIT-II found that although mortality risk increased as a function of time from MI, randomisation to an ICD only conferred a survival benefit in those patients $\geq$ 18 months remote from their qualifying MI (HR $0.55,95 \% \mathrm{CI}$ $0.39-0.78, p=0.001)$ versus those with relatively recent MI ( $<18$ months; HR 0.97, $p=0.92$ ), with this benefit remaining substantial for $\geq 15$ years post-infarct [28].

A further, more troubling possibility is that ICDs might increase non-arrhythmic mortality not merely indirectly, by 'switching' the mode of death, but directly, via intrinsically deleterious mechanisms. This relates less to peri-procedural deaths - of which there was only one in IRIS and none in DINAMIT - but rather the consequences to the myocardium of high voltage defibrillation therapy. Early signals of this possibility were that randomisation to a defibrillator was associated with an increased risk of rehospitalisation in AVID, and with a higher rate of new or worsening heart failure in MADIT II. Subsequent analyses of several ICD trials, including DINAMIT and MADIT II, demonstrated an association between ICD shocks (both appropriate and inappropriate) and ACM $[25,29,30]$. Evidence supporting a causative relationship arrived with the publication of the Multicenter Automatic Defibrillator Implantation Trial-Reduce Inappropriate Therapy (MADIT-RIT) study in 2012, in which randomisation to either of two novel ICD programming modes - both of which reduced appropriate and inappropriate ICD therapy-resulted in lower ACM than with randomisation to conventional ICD programming (which was most similar to the ICD programming used in the RCTs discussed above) $[31 \bullet]$.

The question of who might benefit from receiving an ICD within 40 days of MI is currently being readdressed in the PROTECT-ICD trial (see Table 2). In the intervention arm of the trial, patients within 2-40 days of an MI will undergo 
an EP study, whereby those with inducible VT will receive an ICD, and those without shall not. Participants will alternatively be randomised to a control arm (standard care). In a substudy of the trial, cardiac magnetic resonance (CMR) imaging will be performed, for correlation of CMR data with the presence of inducible VT at EP study and clinical outcomes. The primary endpoint of the trial is SCD or non-fatal arrhythmia, which may garner criticism given the issues discussed above.

\section{Patients Undergoing Coronary Artery Bypass Grafting}

The Coronary Artery Bypass Grafting Patch (CABG-Patch) trial examined whether an ICD could reduce mortality in patients undergoing coronary artery bypass graft (CABG) surgery [6]. Eligible patients were already scheduled for CABG and required to have $\mathrm{LVEF} \leq 35 \%$ and abnormalities on an SAECG. Nine hundred participants were randomised to perioperative implantation of an epicardial ICD or standard care in a 1:1 ratio. During an average follow-up of 32 months, there were 101 deaths from any cause in the ICD group and 95 in the control group (HR 1.07, 95\%CI 0.81-1.42). This was despite a borderline significant reduction in SCD with an ICD (HR 0.55, 95\% CI 0.29-1.03; $p=0.06$ ). Underlying this, SCD comprised just $29 \%$ of all deaths in the control group (compared to, for example, 49\%, 50\% and 51\% in MADIT II, DINAMIT and IRIS respectively). Resultantly, it was more challenging for a defibrillator to significantly reduce SCD, and harder still for any such reduction to affect ACM. The explanation for this low rate of SCD is not certain. However, it was notable that the difference in the rates of SCD between the groups increased with duration of follow-up, with the Kaplan-Meier curves for SCD separating after $\sim 6$ months and diverging progressively over the follow-up period. A possible explanation is that revascularisation at the time of randomisation temporarily negated a potent substrate for arrhythmic death, since it has been estimated that 35-58\% of SCD is preceded by myocardial ischaemia [32, 33]. By contrast, revascularisation was not universal in MADIT II, IRIS or DINAMIT, with a third of patients in DINAMIT receiving no revascularisation following their index MI. The implication is that, without a method to more precisely identify patients at high risk of SCD post-CABG, the rate of fatal VA in this population is too low to warrant a broad indication for a peri-operative defibrillator implantation. Because the rate of SCD increased as a function of time from revascularisation in CABG-Patch - presumably due to the development of graft failure - it would seem logical for a follow-up trial to have examined the potential benefit of an ICD in patients more remote from their index surgical procedure. However, a large proportion of this population was represented in the subsequent MADIT II trial, in which over half of patients had a previous $\mathrm{CABG}$, thus diluting this impetus.

\section{Patients with Heart Failure and Reduced Ejection Fraction (HFrEF)}

The Sudden Cardiac Death in Heart Failure Trial (SCD-HeFT) was the first RCT to examine whether an ICD would reduce the risk of death in a population with chronic heart failure and reduced ejection fraction (HFrEF) of either ischemic or nonischemic aetiology (see Table 1 for trial characteristics) [15•]. The investigators also sought to determine whether the efficacy of an ICD was influenced by heart failure symptom severity. Two thousand five hundred twenty-one patients in NYHA functional classes II to III were randomised to placebo, amiodarone or an ICD. Over a median follow-up of 46 months, ICD treatment reduced ACM by $23 \%$ compared to placebo (HR $0.77,97.5 \%$ CI $0.62-0.96, p=0.007$ ). The survival benefit conferred by an ICD did not differ according to ischemic or non-ischemic aetiology $(P$ for interaction $=0.68)$, implying a survival advantage in patients with NICM. This benefit was maintained for patients in NYHA functional class II (HR 0.54, 97.5\% CI $0.40-0.74, p<0.001$ ), but not for those in NYHA class III ( $P$ for interaction $<0.001)$. One potential explanation for this finding is that the competing risk of pump failure death in NYHA functional class III patients was too substantial for ICDs to reduce overall mortality. This possibility is supported by other studies in which patients with more advanced symptoms were more likely to die from pump failure than SCD [34-36]. However, the HR for SCD and other cardiovascular death (CVD) subtypes stratified by NYHA class were not published, and moreover, this finding was not replicated in MADIT II, in which there was no interaction between treatment effect and NYHA class [37].

The survival benefit of an ICD in relation to NYHA class was examined further in a recent patient-level meta-analysis of four primary prevention trials [38]. For the 1867 patients in NYHA class II, an ICD was associated with a reduction in ACM (HR 0.55, 95\%CI 0.35-0.85). ACM was not significantly reduced for ICD-treated patients in NYHA class III (HR 0.76, 95\%CI, 0.48-1.24); however, there were substantially fewer patients in this subgroup $(n=896)$, and the point estimate suggests a significant effect might have been proven had the group been larger. To add context to this issue, the distinction between NYHA functional classes is subjective, and individual patients within the same NYHA class may be at substantially different risk of sudden and non-sudden death according to other important characteristics, such as LVEF and the presence of severe comorbidity. Appropriately, international guidelines regarding primary prevention ICDs make no overriding distinction between recommendations for patients in NYHA functional class II or III (see Fig. 1). However, it is widely assumed that patients in NYHA class IV are at too high risk of pump failure death to benefit from an ICD. These patients were largely excluded from the above trials. 


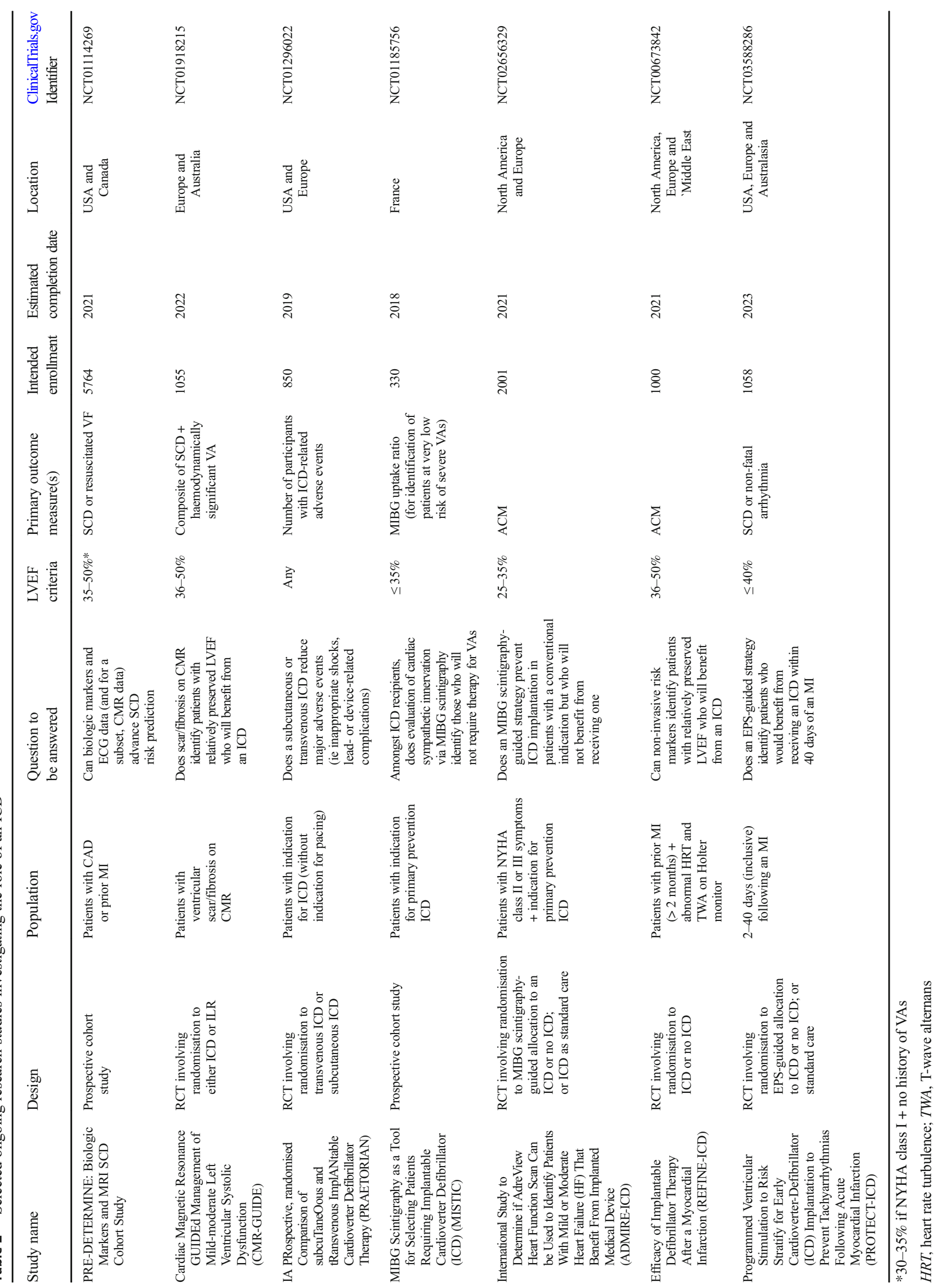


The Comparison of Medical Therapy, Pacing, and Defibrillation in Heart Failure (COMPANION) trial randomised 1520 patients with HFrEF to CMT alone or in combination with either a cardiac resynchronisation pacemaker (CRT-P) or cardiac resynchronisation defibrillator (CRT-D) [14]. Patients were in either NYHA functional classes III or IV, and both ICM and NICM patients were included. Across the trial population, assignment to CRT-D was associated with a $36 \%$ reduction in ACM when compared to CMT (HR 0.64, $95 \%$ CI $0.48-0.86, p=0.003)$. This was driven in part by a $56 \%$ reduction in SCD (HR for CRT-D vs CMT 0.44 , $95 \%$ CI $0.23-0.86, p=0.02$ ). It should be noted that these HRs incorporate the extra mortality benefit of randomisation to CRT in addition to that derived from a defibrillator. Specifically, amongst patients with NICM, CRT-D therapy led to a lower risk of ACM, as compared with CMT (HR $0.50,95 \%$ CI $0.29-0.88$ ). There was no interaction between ischemic/non-ischemic aetiology of HFrEF and the survival benefit of CRT-D over pharmacologic therapy.

\section{Patients with HFrEF and a Presumed Non-ischemic Aetiology: the Pre-DANISH Era}

The first two primary prevention ICD trials (the Cardiomyopathy Trial (CAT) and Amiodarone Vs Implantable Cardioverter-Defibrillator (AMIOVIRT) trial) focussing on non-ischemic cardiomyopathy (NICM) were stopped early for futility, resulting in part from mortality rates that were far lower than anticipated [11, 12]. In CAT, for example, the rate of ACM after 12 months was just 5.6\% in the control group, and although final rates of SCD were not reported, none occurred during the first 2 years. The Defibrillators in Non-Ischemic Cardiomyopathy Treatment Evaluation (DEFINITE) trial subsequently assigned 458 patients with NICM to medical therapy with or without a primary prevention ICD. Assignment to an ICD reduced SCD (HR $0.20,95 \%$ CI $0.06-0.71, p=0.006$ ), but ACM was not significantly different between the groups (HR $0.65, p=0.08$ ) [13]. This was because the incidence of SCD was lower than anticipated, comprising $35 \%$ of all deaths in the control group. Over a mean follow-up of 29 months, there were only 68 deaths from any cause. Results from the SCD-HeFT and COMPANION trials involving subgroups with NICM are discussed above.

The CAT, AMIOVIRT, DEFINITE and SCD-HeFT and COMPANION trials were subsequently combined in a metaanalysis, which was until recently the most robust evidence available regarding the efficacy of ICDs in NICM [39]. Pooled analysis indicated a reduction in ACM amongst patients randomised to a defibrillator (either ICD or CRT-D) when compared to medical therapy (HR 0.69 , 95\% CI $0.55-$ $0.87, p=0.002$ ). It is important to note that the data utilised in this meta-analysis from COMPANION were a comparison of
CRT-D and medical therapy, since the effect of CRT-D versus CRT-P was not available at the time. ${ }^{3}$ Since CRT reduces both SCD and other forms of death in patients with heart failure [40], the perceived efficacy of a defibrillator in this analysis may have been overestimated by the concomitant benefit of CRT.

Despite the lack of an RCT showing outright benefit of an ICD in patients with NICM, current ESC guideline recommendations for primary prevention ICDs in patients with HFrEF (published 2015 and based on MADIT II, SCDHeFT, DEFINITE and the meta-analysis discussed above) vary little according to ischemic/non-ischemic aetiology (see Fig. 1). The ACC/AHA/HRS guidelines were published in 2017, subsequent to the results of the DANISH trial, which is discussed next.

\section{Patients with HFrEF and a Presumed Non-ischemic Aetiology: the DANISH Trial}

The largest and most recent primary prevention ICD trial in patients with NICM was the Danish Study to Assess the Efficacy of ICDs in Patients with Non-ischemic Systolic Heart Failure on Mortality (DANISH) [16•]. DANISH randomised 1116 patients to optimal medical therapy with or without a defibrillator in a 1:1 allocation. After a median follow-up period of 5.6 years, ACM did not differ significantly between the defibrillator and control groups, in which there were 120 and 131 deaths respectively (HR, $0.87, p=0.28$ ), despite the fact that an ICD conferred a $50 \%$ reduction in the risk of SCD (HR 0.50, 95\%CI 0.31-0.82, $p=0.005$ ).

What can we learn from DANISH? In this contemporary trial, SCD constituted $35 \%$ of overall mortality in the control group, matching the proportion found in DEFINITE. However, whilst the annual mortality rate in DEFINITE was low at $7 \%$, it was just $5 \%$ in DANISH. Resultantly, there were only 70 sudden deaths overall, with the rate of SCD in the control group less than $2 \%$ per year. This means the absolute reduction in SCD was just 3.9\% during a follow-up of more than 5 years. More broadly, CVD comprised only $73 \%$ of ACM amongst patients in the control group, a relatively small proportion when set against older ICD trials, and heart failure trials more generally ( $82 \%$ in MADIT II, for instance).

Underlying these low mortality rates, the use of neurohormonal antagonist drugs with prognostic benefit in heart failure was high in DANISH, with angiotensin-converting enzyme (ACE) inhibitors or angiotensin II receptor blockers (ARBs), beta-blockers and mineralocorticoid receptor antagonists (MRAs) prescribed to $97 \%, 92 \%$ and $58 \%$ of patients respectively. In parallel, $58 \%$ of patients in both treatment arms were

\footnotetext{
${ }^{3}$ Recently, a comparison of the CRT-D and CRT-P arms from COMPANION was published [43•]: the $\mathrm{HR}$ for ACM was 0.84 (95\% CI 0.65-1.09), for CVD it was $0.73(95 \% \mathrm{CI} 0.55-0.98)$ and for SCD it was 0.37 (95\%CI $0.21-0.65$ ).
} 
receiving cardiac resynchronisation therapy (CRT). In patients with heart failure, these pharmacologic therapies and CRT each additively reduce the risk of both SCD and pump failure death, and indeed of CVD more generally. As these treatments are increasingly more prevalent, rates of both sudden and cardiovascular death are falling across RCTs enrolling patients with heart failure [41, 42]. Resultantly, in an optimally treated trial population, even a large reduction in SCD may fail to significantly impact overall mortality if SCD is a relatively small proportion of CVD, and CVD is in turn a relatively small proportion of ACM. For trialists seeking to show that an ICD - a treatment which reduces SCD but not other modes of death - confers an overall survival benefit, this increases the challenge.

In this context, recent analysis of patients receiving the most optimal medical therapy in COMPANION yields a salient observation [43•]. Across the entire trial cohort, the reductions in ACM with CRT-D and CRT-P when each was compared to CMT were $36 \%$ and $24 \%$ respectively (CRT-D vs CMT: HR 0.64, 95\%CI 0.48-0.86; CRT-P vs CMT: HR $0.76,95 \%$ CI $0.57-1.01)$. However, amongst patients receiving two neurohormonal antagonists (ACE-inhibitor or ARB, plus beta-blocker; $\sim 61 \%$ of patients in COMPANION), the difference in treatment efficacy between CRT-D and CRT-P was abolished (CRT-D vs CMT: HR 0.56, 95\%CI 0.37-0.85; CRT-P vs CMT: HR 0.57, 95\%CI 0.37-0.85). A similar trend was evident in the much smaller subgroup of patients who were additionally receiving an MRA. The implication is that in the best-treated patients, a defibrillator confers no incremental benefit on top of CRT plus optimal medical therapy. This is supported by a recent meta-analysis of COMPANION and DANISH, in which there was no significant difference in ACM between patients randomised to CRT-P or CRT-D [44].

A declining rate of CVD associated with optimal medical therapy also lends greater weight to the contribution of noncardiovascular death to ACM. Since comorbidity associates with older age, a proportionately greater incidence of noncardiovascular death is liable to emerge in trials with longer follow-up and lower CVD, such as DANISH. In keeping with this, there was a significant interaction between age and the treatment effect of an ICD in DANISH, with benefit suggested for patients in the younger two tertiles, but not in the older tertile: age $<59$ years (HR $0.51,95 \%$ CI $0.29-0.92$ ), age $\geq 59$ to $<68$ years (HR $0.75,95 \%$ CI $0.48-1.16$ ) and $\geq 68$ years $($ HR $1.19,95 \%$ CI $1.19-1.73)(P$ for interaction $=0.009)$. Moreover, the investigators reported that the proportional hazard assumption for ACM was violated ( $p=0.054$ when tested with Schoenfeld residuals), implying that the benefit derived from treatment with an ICD varied as the trial progressed. Reflecting this, the Kaplan-Meier curves diverged during the first 5 years, before converging and eventually crossing after $\sim 7.5$ years. In totality, this suggests that ICDs reduce overall mortality in younger patients before the development of worsening heart failure and/or non-cardiovascular comorbidity, leading to an increasing risk of non-sudden death. A recent pre-specified subgroup analysis by the DANISH investigators further examined the interaction between age and ICD benefit [45]. This demonstrated that with an ICD, each year of younger age was associated with a $3.0 \%(95 \% \mathrm{CI}, 0.03-6.0 ; p=$ 0.03 ) lower relative risk of $\mathrm{ACM}$, with the point estimate crossing unity at $\sim 70$ years of age. When dichotomised, patients $\leq 70$ years of age had significantly better survival with an ICD (HR, $0.70 ; 95 \% \mathrm{CI}, 0.51-0.96 ; p=0.03$ ) whereas those $>70$ years old did not (HR $1.05 ; p=0.84$ ). The rates of sudden and non-sudden death in the control group were 1.8 and 2.7 events per 100 patient-years respectively in those aged $\leq$ 70 years. In contrast, they were 1.6 and 5.4 events per 100 patient-years respectively for those aged $>70$ years.

\section{Where Do We Go From Here?}

The DANISH trial reminds us that the evolution of pharmacologic therapy and CRT has altered the landscape in which trialists seek to prove a survival benefit with ICD therapy. Only where there is a sufficiently high risk of arrhythmic death, and concomitantly low risk of all other forms of death, can the capacity for ICDs to reduce SCD translate into an observable reduction in ACM. But the majority of patients with heart failure and/or LVSD no longer die from arrhythmic causes, and CVD has also proportionally declined, resulting in non-cardiovascular mortality playing an increasingly prominent role. Insights from DANISH affirm that increasing age and the development of comorbidity identify patients at higher risk of non-sudden death i.e. those less likely to benefit from a defibrillator. As time moves on, so competing risks of death emerge. In this regard, the much shorter follow-up periods employed in earlier ICD trials are notable: in MADIT II and COMPANION for instance, the durations of follow-up were just 20 and $\sim 15$ months respectively. Would the benefit of an ICD in these RCTs have been so great had trial follow-up been longer?

This question arises within the broader issue of whether evidence from historic ICD trials remains enduringly robust in the modern era. Across the key trials of both primary and secondary prevention therapy (amongst the latter group of which it should be noted that not all patients had LVSD), prescription rates of ACE-inhibitors, ARBs, beta-blockers and MRAs were either sub-optimal by contemporary standards or not recorded (in which scenario utilisation was likely low). Moreover, sacubitril-valsartan was not yet available and few patients would have received CRT, which was still an investigational device during the enrolment periods of most of these trials. If the RCTs upon which the guidelines are based were repeated today, how might this range of prognostic therapies, appropriately applied, affect the result? Randomised data regarding the benefit of catheter ablation for secondary 
prevention of ventricular arrhythmias are lacking; however, this treatment too would now be available for the control group if the secondary prevention ICD trials were re-run. An updated trial of primary prevention ICDs in patients with ICM will surely be performed at some point. Will anyone be courageous enough to perform a similar trial of secondary prevention ICDs?

A pressing challenge is to better identify patient subgroups likely to benefit from an ICD beyond relatively blunt risk stratifiers such as LVEF, NYHA classification and ischemic aetiology of heart failure. A vast array of parameters has been evaluated without overwhelming success, including laboratory indices and biomarkers, electrocardiographic and functional variables, novel echocardiographic techniques such as global longitudinal strain, tissue characterisation by CMR and special imaging modalities such as Iodine-123 metaiodobenzylguanidine (123 I- $m \mathrm{IBG})$. A selection of ongoing research studies is presented in Table 2. These largely focus on refining patient selection within populations with an existing indication for a defibrillator, or identifying patients who might benefit amongst populations without an existing indication e.g. primary prevention ICDs in patients with LVEF $>35 \%$. In the former category, the challenge will be to sub-select patients with the lowest competing risk of non-sudden mortality amongst those with established high risk of SCD. In the latter category, the competing risk of pump failure death will certainly be lower than in patients with $\mathrm{LVEF} \leq 35 \%$, but will their risk of arrhythmic SCD be sufficiently high? In a recent study of 912 unselected 'sudden' deaths in the United States, rigorous application of World Health Organisation diagnostic criteria for SCD by investigators resulted in the reclassification of $>40 \%$ of deaths as non-sudden. [46] Less than half of presumed SCDs were adjudicated as being of arrhythmic aetiology. This suggests that the capacity of an ICD to reduce sudden deaths amongst populations without an existing indication for a defibrillator may be lower than might otherwise be assumed.

For completeness, it should also be noted that, although relatively rare, proarrhythmic gene defects (e.g. Lamin A/C) and other inherited primary arrhythmia syndromes (e.g. the Brugada and Long or Short QT Syndromes) may occasionally identify patients at sufficiently high risk of SCD to warrant an ICD. Similarly, guideline recommendations exist $[19,20]$ for ICD use in a range of structural heart diseases (e.g. certain congenital heart defects and hypertrophic, restrictive, or arrhythmogenic right ventricular, cardiomyopathies), systemic diseases with cardiac involvement (e.g. sarcoidosis) and several other special populations (such as certain patients with a ventricular assist device or awaiting - or in receipt of - a cardiac transplant). There is a paucity of robust evidence for such niche indications, which are themselves beyond the scope of this review.

A final question is the extent to which further advances in the ICD itself might be important. The development of transvenous systems, anti-tachycardia pacing, biphasic shock generators, improved battery technology (limiting the need for generator replacements), telemetry capability, and most recently the emergence of superior programming configurations, have refined the risk-benefit ratio intrinsic to the devices themselves (as opposed to that conferred by the characteristics of the recipient). Amongst patients in the AVID trial who died suddenly despite an ICD, there was the suggestion that a significant proportion of deaths was attributable to the failure of the defibrillator to terminate a VA [47]. By contrast, in the modern era ICDs abort $\geq 95 \%$ of malignant VAs [48]. Similarly, considering the supposition that treatment with an ICD might have directly increased non-sudden cardiac mortality in earlier ICD trials, such a trend was not detectable in DANISH, which thus far is the only primary prevention trial to have implemented 'MADIT-RIT programming', and consequently reported a comparatively low number of inappropriate shocks. It is unclear how much benefit can be wrought from further technical improvements in the functionality of an ICD, but it seems likely that most of the gains have already been made. The place of alternative ICD delivery systemsnamely subcutaneous devices and non-implanted, wearable devices - remains to be fully elucidated and detailed discussion of them is beyond the remit of this review.

\section{Conclusion}

Which patients stand to benefit from an ICD? At first glance, this would appear to be patients with a high risk of arrhythmic SCD and relatively low risk of other modes of death. This ostensibly leads to the assumption that ICDs must reduce mortality for populations in which these characteristics are observed. However, as suggested in DINAMIT and IRIS, patients who require defibrillator shocks to abort malignant VAs may be at higher risk of other modes of death than apparently similar patients who do not. Therefore, a more robust answer is: those patients with a sufficiently high risk of arrhythmic SCD and sufficiently low risk of all other modes of death even when treated with a defibrillator. The determination of whether an ICD reduces mortality is thus impossible to make outside of a randomised trial. Hazard ratios for ACM in the major primary and secondary prevention RCTs reflect broad populations fitting this description. However, scrutiny of the evidence affirms that decisions regarding an ICD should be tailored to individual risk, regardless of whether that person meets the wider inclusion criteria of a positive trial. Consideration should be made, for example, of age, aetiology of heart failure and/or presence of CAD, history of syncope, NYHA functional class, severity of comorbidity, tolerated pharmacologic therapy and the presence of - or suitability for-CRT. To differing degrees, this premise holds true regardless of the indication for a defibrillator. The complexity 
of this situation has been compounded by the revolution in prognostic therapies for LVSD with or without heart failure over the past three decades, which means that the absolute validity of historical trial evidence is open to question. It is increasingly obvious that new RCTs are necessary, and the possible variations upon these are extensive [49]. What remains to be seen is how far trial investigators will dare to go in re-challenging the evidence base.

\section{Compliance with Ethical Standards}

Conflict of Interest Simon AS Beggs reports personal fees and nonfinancial support from Abbott and personal fees from Vifor, outside the submitted work.

Roy Gardner reports personal fees and non-financial support from Abbott, personal fees and non-financial support from Boston Scientific, personal fees and non-financial support from Novartis and personal fees and non-financial support from Vifor, outside the submitted work.

John McMurray reports other from GlaxoSmithKline, during the conduct of the study; other from Bayer, other from Cardiorentis, other from Amgen, other from Oxford University/Bayer, other from Theracos, other from Abbvie, other from DalCor, other from Pfizer, other from Merck, other from AstraZeneca, other from Bristol Myers Squibb (BMS), other from Kidney Research UK (KRUK)/Kings College Hospital, London/ Vifor-Fresenius Pharma and other from Novartis, outside the submitted work.

Human and Animal Rights and Informed Consent This article does not contain any studies with human or animal subjects performed by any of the authors.

Open Access This article is distributed under the terms of the Creative Commons Attribution 4.0 International License (http:// creativecommons.org/licenses/by/4.0/), which permits unrestricted use, distribution, and reproduction in any medium, provided you give appropriate credit to the original author(s) and the source, provide a link to the Creative Commons license, and indicate if changes were made.

\section{References}

Papers of particular interest, published recently, have been highlighted as:

- Of importance

1. Mirowski M, Reid PR, Mower MM, Watkins L, Gott VL, Schauble JF, et al. Termination of malignant ventricular arrhythmias with an implanted automatic defibrillator in human beings. N Engl J Med. 1980;303:322-4.

2. The Antiarrhythmic Versus Implantable Defibrillators (AVID) investigators. A comparison of antiarrhythmic-drug therapy with implantable defibrillators in patients resuscitated from near-fatal ventricular arrhythmias. N Engl J Med. 1997;337:1576-83 This landmark study is the largest and arguably the most important of the secondary prevention defibrillator trials.

3. Kuck KH, Cappato R, Siebels J, et al. Randomized comparison of antiarrhythmic drug therapy with implantable defibrillators in patients resuscitated from cardiac arrest: the Cardiac Arrest Study Hamburg (CASH). Circulation. 2000;102:748-54.
4. Connolly SJ, Gent M, Roberts RS, Dorian P, Roy D, Sheldon RS, et al. Canadian Implantable Defibrillator Study (CIDS): a randomized trial of the implantable cardioverter defibrillator against amiodarone. Circulation. 2000;101:1297-302.

5. Moss AJ, Hall WJ, Cannom DS, Daubert JP, Higgins SL, Klein H, et al. Improved survival with an implanted defibrillator in patients with coronary disease at high risk for ventricular arrhythmia. $\mathrm{N}$ Engl J Med. 1996;335(26):1933-40.

6. Bigger JT, Whang W, Rottman J, et al. Mechanisms of death in the $\mathrm{CABG}$ patch trial. A randomized trial of implantable cardiac defibrillator prophylaxis in patients at high risk of death after coronary artery bypass graft surgery. Circulation. 1999;99:1416-21.

7. Buxton AE, Lee KL, Fisher JD, Josephson ME, Prystowsky EN, Hafley G. A randomized study of the prevention of sudden deaths in patients with coronary artery diease. $\mathrm{N}$ Engl J Med. 1999;341(25):1882-90.

8. Moss AJ, Zareba W, Hall WJ, et al. Prophylactic implantation of a defibrillator in patients with myocardial infarction and reduced ejection fraction. N Engl J Med. 2002;346(12):877-83 MADIT II is the seminal trial examining the use of an ICD as a primary prevention therapy in a population with previous myocardial infarction.

9. Hohnloser SH, Kuck KH, Dorian P, et al. Prophylactic use of an implantable cardioverter-defibrillator after acute myocardial infarction. N Engl J Med. 2004;351(24):2481-8 The DINAMIT trial exemplifies why an appreciation of competing modes of death is critical in the evaluation of a defibrillator in a clinical trial, and demonstrates why a reduction in sudden death alone is insufficient basis to recommend the use of an ICD.

10. Steinbeck G, Andresen D, Seidl K, et al. Defibrillator implantation early after myocardial infarction. N Engl J Med. 2009;361(15): 1427-36 As for DINAMIT, so for IRIS.

11. Bänsch D, Antz M, Boczor S, et al. Primary prevention of sudden cardiac death in idiopathic dilated cardiomyopathy: the Cardiomyopathy trial (CAT). Circulation. 2002;105:1453-8.

12. Strickberger SA, Hummel JD, Bartlett TG, Frumin HI, Schuger $\mathrm{CD}$, Beau SL, et al. Amiodarone versus implantable cardioverterdefibrillator:randomized trial in patients with nonischemic dilated cardiomyopathy and asymptomatic nonsustained ventricular tachycardia-AMIOVIRT. J Am Coll Cardiol. 2003;41:1707-12.

13. Kadish A, Dyer A, Daubert JP, Quigg R, Estes NA, Anderson KP, et al. Prophylactic defibrillator implantation in patients with nonischemic dilated cardiomyopathy. N Engl J Med. 2004;350: 2151-8.

14. Bristow MR, Saxon LA, Boehmer J, Krueger S, Kass DA, de Marco T, et al. Cardiac-resynchronization therapy with or without an implantable defibrillator in advanced chronic heart failure. $\mathrm{N}$ Engl J Med Overseas Ed. 2004;350:2140-50.

15. Bardy GH, Lee KL, Mark DB, et al. Amiodarone or an implantable cardioverter defibrillator for congestive heart failure. $\mathrm{N}$ Engl J Med. 2005;352:225-37 SCD-HeFT extended the evidence base for use of a primary prevention ICD to patients with chronic symptomatic heart failure.

16. Køber L, Thune JJ, Nielsen JC, et al. Defibrillator implantation in patients with nonischemic systolic heart failure. N Engl J Med. 2016;375:1221-30 The DANISH trial is the largest and most contemporary trial of defibrillator therapy in NICM, and has renewed the debate regarding who benefits from an ICD.

17. Connolly SJ, Hallstrom AP, Cappato R, et al. Meta-analysis of the implantable cardioverter defibrillator secondary prevention trials. AVID, CASH and CIDS studies. Antiarrhythmics vs Implantable Defibrillator study. Cardiac Arrest Study Hamburg. Canadian Implantable Defibrillator Study. Eur Heart J. 2000;21:2071-8 This meta-analysis combines data from the three major trials to examine an ICD as a secondary prevention therapy. 
18. Lee DS, Green LD, Liu PP, Dorian P, Newman DM, Grant FC, et al. Effectiveness of implantable defibrillators for preventing arrhythmic events and death: a meta-analysis. J Am Coll Cardiol. 2003;41(9): 1573-82.

19. Priori SG, Blomstrom-Lundqvist C, Mazzanti A, et al. ESC guidelines for the management of patients with ventricular arrhythmias and the prevention of sudden cardiac death. The task force for the management of patients with ventricular arrhythmias and the prevention of sudden cardiac death of the European Society of Cardiology (ESC). Eur Heart J. 2015;36:2757-9.

20. Al-Khatib SM, Stevenson WG, Ackerman MJ et al. 2017 AHA/ ACC/HRS guideline for management of patients with ventricular arrhythmias and the prevention of sudden cardiac death. A report of the American College of Cardiology/American Heart Association task force on clinical practice guidelines and the Heart Rhythm Society. Circulation. 2017. https://doi.org/10.1161/CIR. 0000000000000549 .

21. Rouleau JL, Talajic M, Sussex B, Potvin L, Warnica W, Davies RF, et al. Myocardial infarction patients in the $1990 \mathrm{~s}$ - their risk factors, stratification and survival in Canada: the Ca- nadian assessment of myocardial infarction (CAMI) study. J Am Coll Cardiol. 1996;27:1119-27.

22. Tavazzi L, Volpi A. Remarks about postinfarction prognosis in light of the experience with the Gruppo Italiano per lo Studio della Sopravvivenza nell' Infarto Miocardico (GISSI) trials. Circulation. 1997;95:1341-5.

23. Cerqueira MD, Maynard C, Ritchie JL, Davis KB, Kennedy JW. Long-term survival in 618 patients from the Western Washington Strepto- kinase in Myocardial Infarction trials. J Am Coll Cardiol. 1992;20:1452-9.

24. de Vreede-Swagemakers JJM, Gorgels APM, Dubois-Arbouw WI, van Ree JW, Daemen MJAP, Houben LGE, et al. Out-of-hospital cardiac arrest in the 1990s: a population-based study in the Maastricht area on incidence, characteristics and survival. J Am Coll Car- diol. 1997;30:1500-5.

25. Moss AJ, Greenberg H, Case RB, Zareba W, Hall WJ, Brown MW, et al. Long-term clinical course of patients after termination of ventricular tachyarrhythmia by an implanted defibrillator. Circulation. 2004;110:3760-5.

26. Camm AJ, Pratt CM, Schwartz PJ, al-Khalidi HR, Spyt MJ, Holroyde MJ, et al. Mortality in patients after a recent myocardial infarction: a randomized, placebo-controlled trial of azimilide using heart rate variability for risk stratification. Circulation. 2004;109:990-6.

27. Buccelletti E, Gilardi E, Scaini E, Galiuto L, Persiani R, Biondi A, et al. Heart rate variability and myocardial infarction: systematic literature review and meta-analysis. Eur Rev Med Pharmacol Sci. 2009;13:299-307.

28. Wilber DJ, Zareba W, Jackson Hall W, et al. Time dependence of mortality risk and defibrillator benefit after myocardial infarction. Circulation. 2004;109:1082-4.

29. Poole JE, Johnson GW, Hellkamp AS, Anderson J, Callans DJ, Raitt MH, et al. Prognostic importance of defibrillator shocks in patients with heart failure. N Engl J Med. 2008;359:1009-17.

30. Dorian P, Hohnloser SH, Thorpe KE, Roberts RS, Kuck KH, Gent M, et al. Mechanisms underlying the lack of effect of implantable cardioverter-defibrillator therapy on mortality in high-risk patients with recent myocardial infarction: insights from the Defibrillation in Acute Myocardial Infarction Trial (DINAMIT). Circulation. 2010;122:2645-52.

31. Moss AJ, Schuger C, Beck CA, et al. Reduction in inappropriate therapy and mortality through ICD programming. N Engl J Med. 2012;367:2275-83. https://doi.org/10.1056/NEJMoa1211107 MADIT-RIT demonstrated that novel defibrillator programming configurations reduce both appropriate and inappropriate ICD therapies, and overall mortality.

32. Greene HL, Richardson DW, Barker AH, Roden DM, Capone RJ, Echt DS, et al. Classification of deaths after myocardial infarction as arrhythmic or nonarrhythmic (the cardiac arrhythmia pilot study). Am J Cardiol. 1989;63:1-6.

33. Marcus FI, Cobb LA, Edwards JE, Kuller L, Moss AJ, Bigger JT Jr, et al. Mechanism of death and prevalence of myocardial ischemic symptoms in the terminal event after acute myocardial infarction. Am J Cardiol. 1988;61:8-15.

34. Effect of metoprolol $\mathrm{CR} / \mathrm{XL}$ in chronic heart failure: Metoprolol CR/XL Randomised Intervention Trial in Congestive Heart failure (MERIT-HF). Lancet. 1999;353:2001-7.

35. Solomon SD, Wang D, Finn P, Skali H, Zornoff L, McMurray JJV, et al. Effect of candesartan on cause-specific mortality in heart failure patients: the Candesartan in Heart failure Assessment of Reduction in Mortality and Morbidity (CHARM) program. Circulation. 2004;110:2180-3.

36. McMurray JJ, Packer M, Desai AS, Gong J, Lefkowitz MP, Rizkala $\mathrm{AR}$, et al. Angiotensin-neprilysin inhibition versus enalapril in heart failure. N Engl J Med. 2014;371:993-1004.

37. Biton Y, Rosero S, Moss A, Zareba W, Kutyifa V, Baman J, et al. Long-term survival with implantable cardioverter-defibrillator in different symptomatic functional classes of heart failure. J Am Coll Card. 2018;121(5):615-20.

38. Friedman DJ, Al-Khatib S, Zeitler EP, et al. New York Heart Association class and the survival benefit from primary prevention implantable cardioverter defibrillators: a pooled analysis of 4 randomized controlled trials. Am Heart J. 2017;191:21-9.

39. Desai AS, Fang JC, Maisel WH, Baughman KL. Implantable defibrillators for the prevention of mortality in patients with nonischemic cardiomyopathy: a meta-analysis of randomized controlled trials. JAMA. 2004;292:2874-9.

40. Cleland JGF, Daubert JC, Erdmann E, Freemantle N, Gras D, Kappenberger L, et al. The effect of cardiac resynchronisation on morbidity and mortality in heart failure. N Engl J Med. 2005;352(15):1539-49.

41. Rush CJ, Campbell RT, Jhund PS, Connolly EC, Preiss D, Gardner RS, et al. Falling cardiovascular mortality in heart failure with reduced ejection fraction and implications for clinical trials. JACC Heart Fail. 2015;3:603-14.

42. Shen L, Jhund PS, Petrie MC, Claggett BL, Barlera S, Cleland JGF, et al. Declining risk of sudden death in heart failure. N Engl J Med. 2017;377:41-51. https://doi.org/10.1056/NEJMoa1609758.

43. Bristow MR, Saxon LA, Feldman AM, et al. Lessons learned and insights gained in the design, analysis, and outcomes of the COMPANION trial. JACC Heart Fail. 2016;4:521-35 Important recent insights into the COMPANION trial.

44. Beggs SAS, Jhund PS, Jackson CE, McMurray JJV, Gardner RS. Non-ischaemic cardiomyopathy, sudden death and implantable defibrillators: a review and meta-analysis. Heart. 2018;104:144-50.

45. Elming MB, Nielsen JC, Haarbo J, Videbæk L, Korup E, Signorovitch J, et al. Age and outcomes of primary prevention implantable cardioverter-defibrillators in patients with nonischemic systolic heart failure. Circulation. 2017;136:1772-80.

46. Tseng ZH, Olgin JE, Vittinghoff E, Ursell PC, Kim AS, Sporer K, et al. Prospective countywide surveillance and autopsy characterization of sudden cardiac death. Circulation. 2018;137:2689-700. https://doi.org/10.1161/CIRCULATIONAHA.117.033427.

47. Investigators AVID. Causes of death in the Antiarrhythmics Versus Implantable Defibrillators (AVID) Trial. J Am Coll Cardiol. 1999;34:1552-9.

48. Healey JS, Hohnloser SH, Glikson M, on behalf of the Shockless IMPLant Evaluation (SIMPLE) investigators, et al. Cardioverter defibrillator implantation without induction of ventricular fibrillation: a single-blind, non-inferiority, randomized controlled trial (SIMPLE). Lancet. 2015;385:785-91.

49. Petrie MC, Connelly DT, Gardner RS. Who needs an implantable cardioverter-defibrillator? Controversies and opportunities after DANISH. Eur J Heart Fail. 2018;20(3):413-6. 